\title{
Device for Ambulatory Diagnosis of Pet's Cardiovascular System Functional State
}

Vitaliy Petrov ${ }^{1}$, Andrey Shestakov ${ }^{2}$, Danil Borchevkin ${ }^{1}$, Stepan Botman ${ }^{1}$, Evgeny Bogdanov ${ }^{1 *}$, Vitaliy Kasymov ${ }^{1}$, Maksim Patrushev $^{1}$ and Natalia Shusharina $^{1}$

${ }^{1}$ Immanuel Kant Baltic Federal University, Aleksandra Nevskogo, 14к2, Kaliningrad, Kaliningrad Oblast-238300, Russia

${ }^{2}$ Research Center of Veterinary and Zoological Engineering of Kaliningrad State Technical University, Russia

"Corresponding author: Evgeny Bogdanov, Immanuel Kant Baltic Federal University, Aleksandra Nevskogo, 14K2, Kaliningrad, Kaliningrad Oblast-238300, Russia, Tel: +7 401 259-55-96; E-mail: eubogdanov@gmail.com

Rec date: Sep 25, 2015; Acc date: Nov 04, 2015; Pub date: Nov 06, 2015

Copyright: ( 2015 Petrov V, et al. This is an open-access article distributed under the terms of the Creative Commons Attribution License, which permits unrestricted use, distribution, and reproduction in any medium, provided the original author and source are credited.

\begin{abstract}
This paper contains a description of device for ambulatory diagnosis of pet's cardiovascular system functional state capable of measuring pulse wave parameters and storing obtained data. One of the main advantages of developed device practical application is elimination of animal anxiety caused by visit to veterinary clinic. The animal during the measurement does not experience any anxiety and stress. The dog's cardiovascular system measurement result was obtained without the stress influence on the animals. This allows to significantly reducing measured pulse wave data misinterpretation. Easy handling of the device and capability of transferring data to external flash drives allows pet owner to make measurements domiciliary and pass the results to veterinarian during the next animal clinic visit.
\end{abstract}

Keywords Cardiovascular diseases; Health monitoring systems; Photoplethysmography; Pulse wave; Remote diagnostics

\section{List of abbreviations used}

CVS-Cardiovascular system; ECG-Electrocardiography; IRInfrared

\section{Background}

At the present time in veterinary medicine electrocardiography (ECG) is widely used for pet's cardiovascular system (CVS) state diagnostics [1]. Although bioelectric potentials recording procedure is rather easy to conduct, for an animal's (dogs) it is still a source of anxiety and stress. Animal stress is an extreme stimulus induced state of body, which leads to activation of nonspecific adaptive mechanisms of the organism. Hunting dog breeds, toy dog breeds and stud breed of cats are more amenable to stress. As any factor that can potentially inflict harm, stress have negative impact on body functions $[2,3]$. These factors also include injury, disease and jitter. Generally, dogs stress is preceded by fear that essentially is the imminent danger caused emotional state which manifests physiological and behavioral responses. Fear always has a specific source and it is always accompanied by changes in the body: palpitation, salivation, shelter seeking. There are two common sources of fear for dogs visiting veterinary: unfamiliar and thus high-stress environment inside veterinary clinic and negative memories occasioned by veterinary procedure dog undergone in the past. Dog can be irritated by strong odor common for clinics, other animals and nervousness of surrounding persons. As a consequence, animal experience significant changes of muscular tone, blood pressure and temperature, which makes it impossible to conduct cardiovascular system state estimation procedures in veterinary clinic due to lack of accuracy and reliability of obtained data. The fact that physiological parameters change significantly under the influence of stress caused by unfamiliar surroundings and external irritable factors (unusual placement, smells, strangers) leads to difficulties with interpretation of ECG data. Thus, establishing diagnosis in addition to ECG also require full cardiological examination, which include physical diagnosis, echocardiography (ultrasound of the heart), chest radiography and biochemical analysis of blood.

\section{Ethic statement}

All experiments with the dogs were carried out in strict accordance with the directive of the Ministry of Health and Social Development of the Russian Federation dated 23 August 2010 \#708n "On approval of the laboratory practices". The protocol was approved by the Committee on the Ethics of Animal Experiments of the Immanuel Kant Baltic Federal University (Order Number: 347-12).

\section{Methods}

This paper describes cardiac parameters estimation method based on optical measurement of infrared (IR) light absorbed by tissues [4]. Dog's body tissues absorb infrared light differently [5]. Soft tissues IR light absorption rate mainly depends on the volume of blood it contains. Real-time measurements of intensity changes in the light which passes through the tissues allow to obtain data concerning cardiovascular system state (heart rate, pulse wave parameters, oxygenation etc.) [6]. Light intensity changes measurement setup include emitting IR diode and photo detector placed on opposite sides of the tissue facing directly at each other. Distance between diode and detector should not be too large in order to prevent signal intensity from approaching detection threshold. The amplitude of resulting periodic signal increases with higher ratio of blood volume to full tissue volume illuminated by IR diode (Figure 1). 
Citation: Petrov V, Shestakov A, Borchevkin D, Botman S, Bogdanov E, et al. (2015) Device for Ambulatory Diagnosis of Pet's Cardiovascular System Functional State. J Veterinar Sci Technol 6: 263. doi:10.4172/2157-7579.1000263

Page 2 of 4

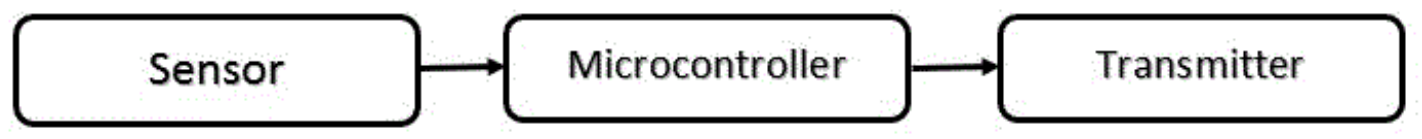

Figure 1: Block diagram of the CVS parameters monitoring device.

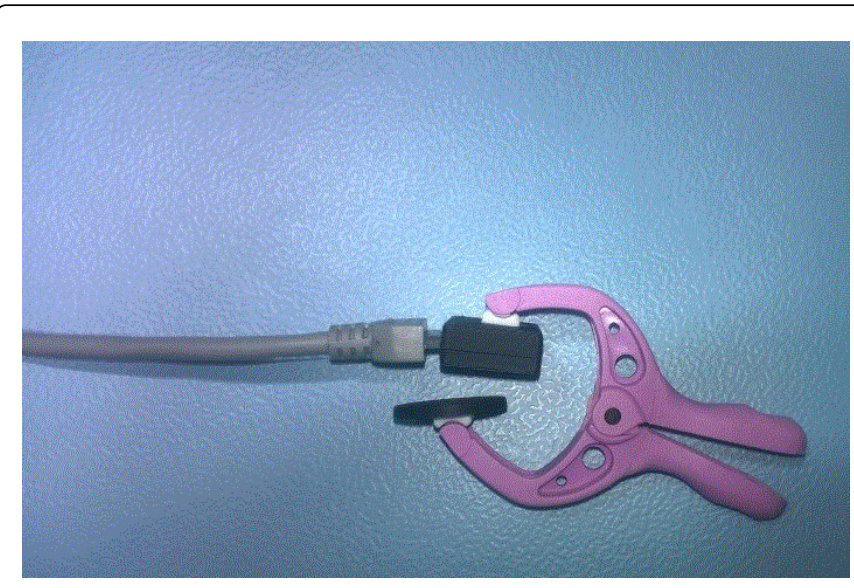

Figure 2: Appearance of device for cardiovascular system functional state diagnostics.

Obviously, optimal place for the signal detection is an ear because it has relatively small thickness and high vessels density. Furthermore, such a choice of detection site allows adapting clip form-factor for the monitoring device making it possible to perform measurements for people without any special veterinary training such as owner of the animal (Figures 2 and 3 ).

Obtained in a comfortable, normal environment for the animal, data then can be sent to a specialist for detailed analysis and establishing diagnosis.

\section{Results and Discussion}

Approbation of the device was performed on three dogs of the same breed (dachshund). Figure 4 shows an image of the original signal received from the device.

In order to filter the signal noise the program was written in the Python 2.7 programming language. This program is based on moving average method, given by the following formula:

$$
S_{t}=\frac{1}{n} \sum_{i=0}^{n-1} m_{t-n}
$$

Number $\mathrm{n}$ of sampling points was determined empirically and amounted to 100 for the first iteration and 50 for the second iteration.

Figures 5-7 presents averaged data obtained from 3 different dogs during one month.

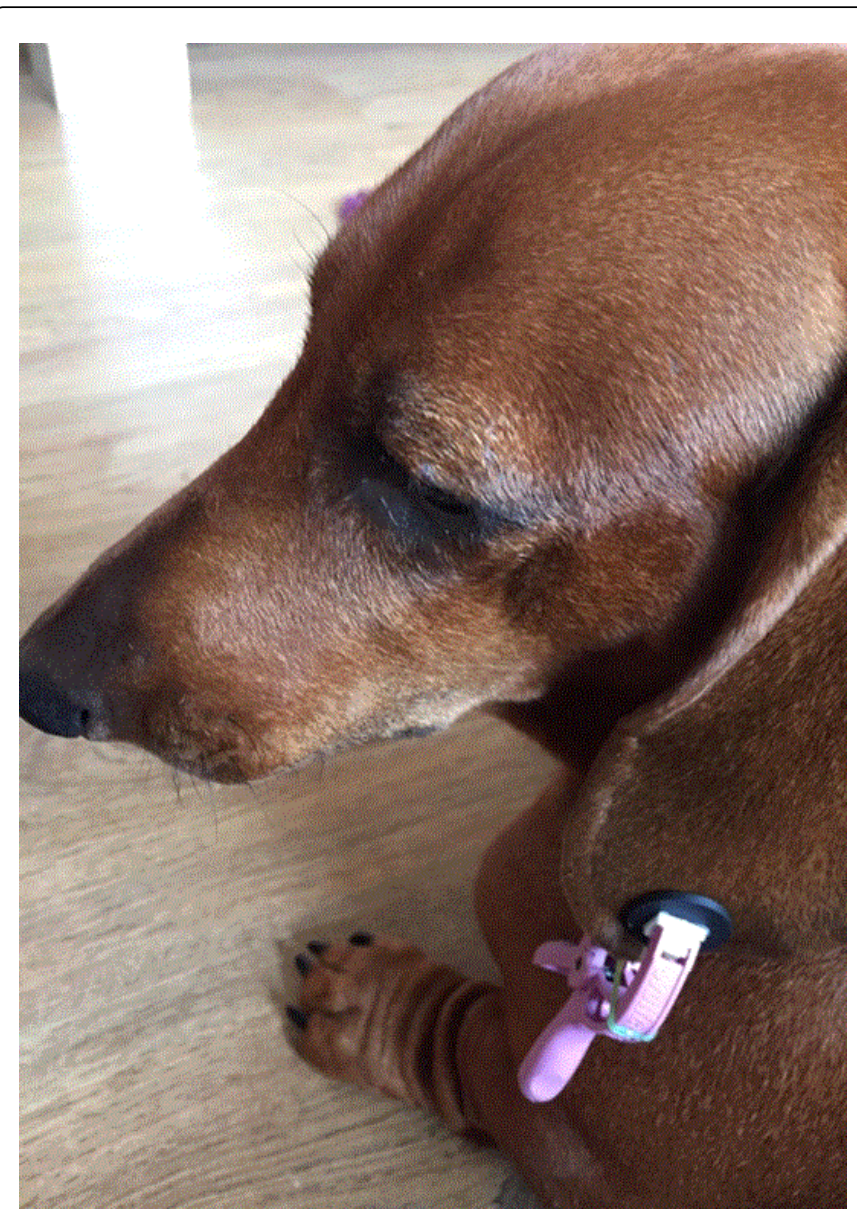

Figure 3: Detection site on the dog's body.

Data handling was carried out using NumPy library, which provide tools for large data sets mathematical processing.

Signal received from the device can be divided into two phases: anacrotic phase expressed by ascending pulse wave branch, and catacrotic phase expressed by descending pulse wave branch, as shown in Figure 8, where $\mathrm{x}$ is amplitude of the systolic peak, and $\mathrm{y}$ is amplitude of the diastolic peak. 
Citation: Petrov V, Shestakov A, Borchevkin D, Botman S, Bogdanov E, et al. (2015) Device for Ambulatory Diagnosis of Pet's Cardiovascular

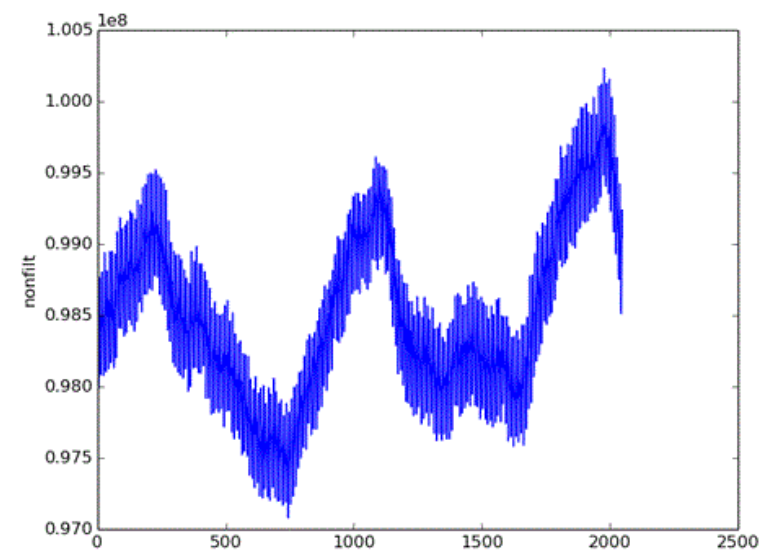

Figure 4: Raw pulse wave data (no filtering performed).

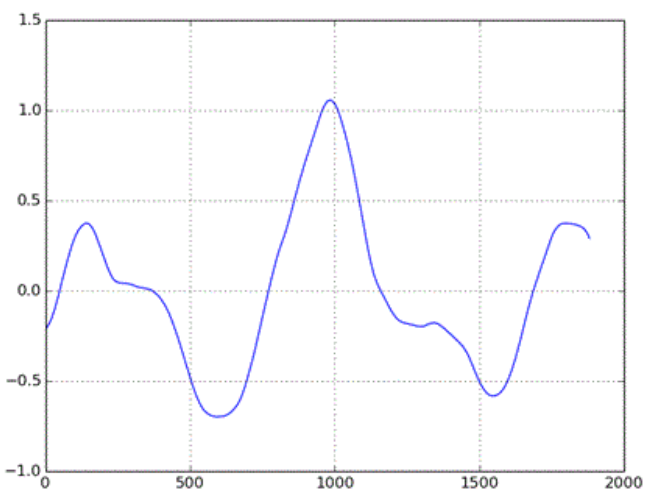

Figure 5: Pulse wave data (first dog).

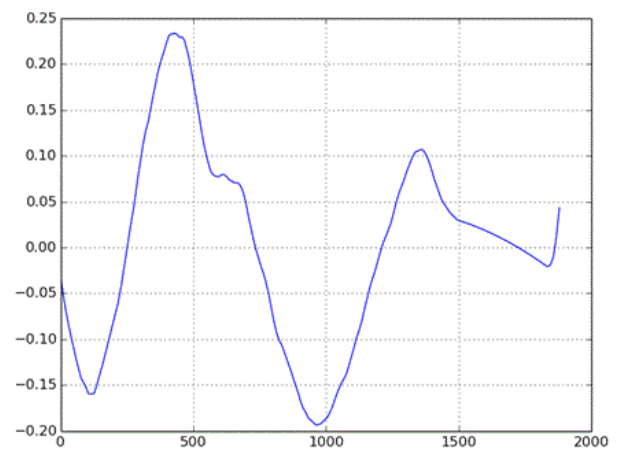

Figure 6: Pulse wave data (second dog).

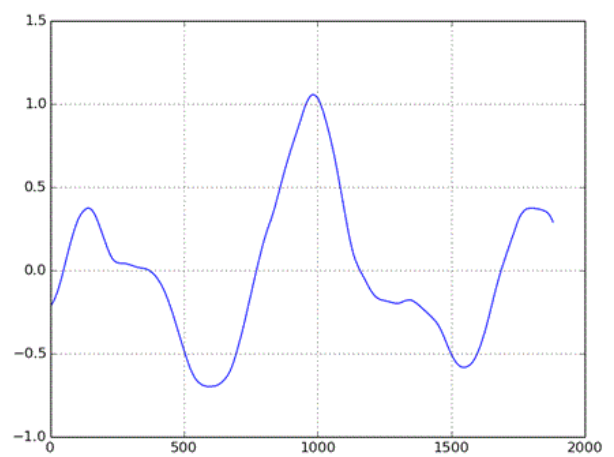

Figure 7: Pulse wave data (third dog).

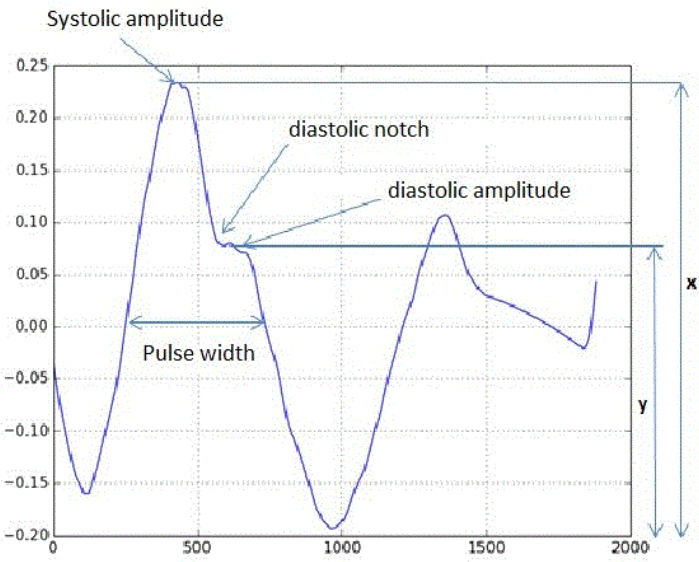

Figure 8: Pulse wave curve characteristic parameters.

The first phase corresponds to the systole and the second one-to diastole and periphery artery wave reflections. Dicrotic notch depicted in Figure 8 is usually observed in catacrotic phase of animals with healthy vessels. Several characteristics can be distinguished:

1) Systolic amplitude: Systolic amplitude (x) illustrated in Figure 7 is quite an important indicator of the pulsating changes of blood volume caused by arterial blood flow around the measuring site. Systolic amplitude is correlated with systolic discharge and is directly proportional to the vascular distensibility in a fairly wide range of cardiac output. It also has been suggested that the systole amplitude is more convenient for blood pressure evaluation than pulse wave propagation time.

2) Pulse width: Figure 8 displays pulse width of the curve. The pulse width is width of the curve at half height of the systolic peak. Pulse width correlates with systemic vascular resistance better than systolic amplitude. 
3) Pulse area: Pulse area is measured as total area under the curve. Pulse area can be divided into two zones by dicrotic notch. The ratio of these two areas illustrated in Figure 9 can be used as indicator of the peripheral vascular resistance.

Inflection point area ratio (IPA) is defined as ratio of areas A2 and A1.

$$
I P A=\frac{A 2}{A 1}(2)
$$

Augmentation index (AI) is defined as the ratio of $\mathrm{y}$ to $\mathrm{x}$ as follows:

$$
A I=\frac{y}{x}(3)
$$

As demonstrated in Figure 8, $\mathrm{x}$ and $\mathrm{y}$ are heights of early and late systolic peak respectively.

4) Inter-systolic interval: The distance between the two nearest systolic peaks is the inter-systolic interval as shown in Figure 10.

5) Total pulse wave time: The distance between the beginning and end of the pulse wave curve is the total pulse wave time as illustrated in Figure 10. The total pulse wave time is usually used instead of intersystolic interval when the diastolic peaks are better expressed and easier to distinguish than systolic ones.

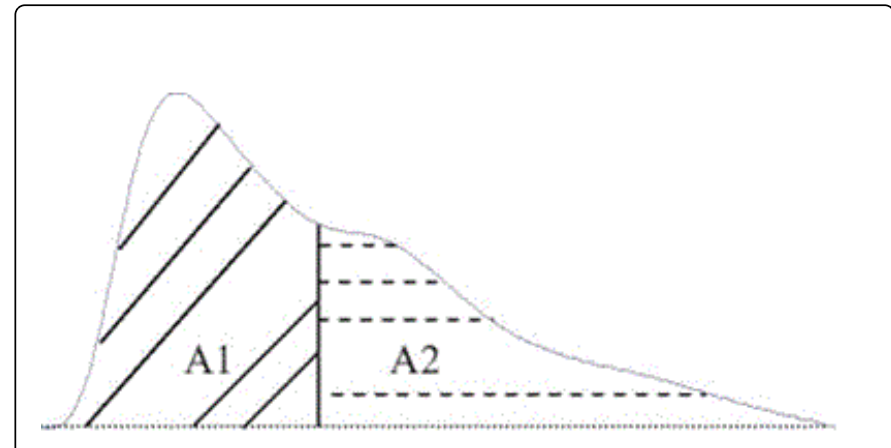

Figure 9: Initial curve. $\mathrm{A} 1$ and $\mathrm{A} 2$ are areas under the curve, separated by the inflection point.

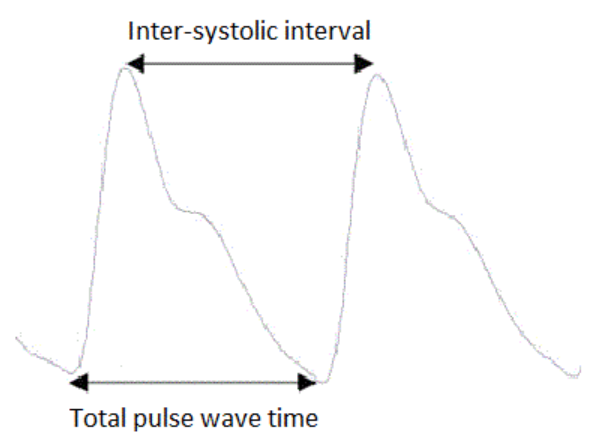

Figure 10: Two consecutive pulse wave peaks.
Currently, there is luck of understanding about diagnostic significance of the different pulse wave parameters and this area requires further research. Occasionally the same indicators are used to measure different but related physiological states.

\section{Conclusion}

The device for ambulatory diagnosis of pet's cardiovascular system functional state was designed and implemented. It was shown that received signal can be used for pulse wave parameters and heart rate calculation.

\section{Competing Interests}

The authors declare that they have no competing interests.

\section{Author's Contributions}

VP, DB, EB participated in study design and coordination, and drafted the manuscript. SB drafted the manuscript. AS directed biological analysis. MP, NS directed the study. All authors read and approved the manuscript.

\section{Acknowledgment}

This work was supported by The Ministry of Education and Science of the Russian Federation grant RFMEFI57814X0053.

\section{References:}

1. Oyama MA, Kraus MS, Gelzer AR (2013) Rapid review of ECG interpretation in small animal practice. CRC Press pp: 43-85.

2. Hekman JP, Karas AZ, Sharp CR (2014) Psychogenic stress in hospitalized dogs: cross species comparisons, implications for health care, and the challenges of evaluation. Animals (Basel) 4: 331-347.

3. Thomason JD, Thomason SS, Fallaw T, Calvert CA, Wolfe HA (2015) ECG of the Month. Journal of the American Veterinary Medical Association 246(9): 962-964.

4. Wilson BC, Jacques SL (1990) Optical reflectance and transmittance of tissues: principles and applications. Quantum Electronics 26: 2186-2199.

5. Duck FA (2013) Physical properties of tissues: a comprehensive reference book. Academic press: pp: 251-320.

6. Bergamasco L, Osella MC, Savarino P, Larosa G, Ozella L et al. (2010) Heart rate variability and saliva cortisol assessment in shelter dog: Human-animal interaction effects. Applied animal behaviour science 125: 56-68. 